\title{
Intensity-dependent effect of treadmill running on rat Achilles tendon
}

\author{
SHAO-YONG XU ${ }^{1}$, YONG-BIN HE ${ }^{1}$, SONG-YUN DENG ${ }^{1}$, SHENG-YAO LIU $^{1}$, LEI XU ${ }^{1}$ and GUO-XIN NI ${ }^{1,2}$ \\ ${ }^{1}$ Department of Orthopaedics and Traumatology, Nanfang Hospital, Southern Medical University, Guangzhou, \\ Guangdong 510515; ${ }^{2}$ Department of Rehabilitation Medicine, First Affiliated Hospital, \\ Fujian Medical University, Fuzhou, Fujian 350005, P.R. China
}

Received December 7, 2015; Accepted January 13, 2017

DOI: $10.3892 /$ etm.2018.6084

\begin{abstract}
It is understood that mechanical loading may affect tendon properties. However, how different mechanical loading conditions may affect tendons remains unknown. The present study aimed to investigate the effect of treadmill running at various intensities on rat Achilles tendon. A total of 18 male Wistar rats were randomly assigned to one of three groups: Control (CON), medium-intensity running (MIR), and high-intensity running (HIR). Following 8 weeks of treadmill running protocols, all Achilles tendons were harvested for histological observation and gene expression analysis. Significant morphological changes were observed with regular and large diameter collagen fibrils in the MIR group, whereas irregular and small diameter collagen fibrils were observed in the HIR group. Collagen type I was significantly upregulated in the MIR group compared with the CON group, and downregulated in the HIR group compared with the CON or MIR groups $(\mathrm{P}<0.05)$. However, collagen type III was significantly upregulated in the HIR group in comparison with the CON or MIR groups $(\mathrm{P}<0.05)$. Furthermore, the expression of matrix metallopeptidase-13 was significantly increased in the MIR and HIR groups compared with the CON group $(\mathrm{P}<0.05)$. The expression of tissue inhibitor of metalloproteinases-1 was increased in the MIR group compared with the CON group, but decreased in the HIR group compared with the CON and MIR groups $(\mathrm{P}<0.05)$. Additionally, decorin expression was significantly higher in the MIR group compared with the CON group, and significantly decreased in the HIR group compared with the CON or MIR groups $(\mathrm{P}<0.05)$. A converse pattern of changes in biglycan expression was identified among the three groups. Aggrecan expression was significantly higher
\end{abstract}

Correspondence to: Professor Guo-Xin Ni, Department of Orthopaedics and Traumatology, Nanfang Hospital, Southern Medical University, 1838 Guangzhou Avenue, Guangzhou, Guangdong 510515, P.R. China

E-mail: guoxinni15tg@163.com

Key words: collagen, proteoglycans, metalloproteinase-13, tissue inhibitor of metalloproteinases-1, rat Achilles tendon in the HIR group compared with the CON or MIR groups $(\mathrm{P}<0.05)$. These findings indicated that moderate exercise may induce increased collagen synthesis and organize regular and large collagen fibers, thus benefiting the Achilles tendon. However, overuse during exercise may result in collagen degradation and disturbance, which predisposes individuals to injury.

\section{Introduction}

The primary function of a tendon is to transmit a mechanical force to move and stabilize joints (1). A tendon is characterized by a relatively small number of tenocyte cells and an extensive extracellular matrix (ECM), which includes collagens, proteoglycans (PGs), glycoproteins and water (2). Its dry mass is composed of $\sim 86 \%$ collagen, $1-5 \%$ PGs and $0.2 \%$ inorganic components (3).

In normal tendons, $90 \%$ of collagen is collagen type I ( Col I) and the second most abundant is collagen type III (Col III) (4). Col I fibers are predominant structural components with dense, parallel arrays, and are regarded as major contributors in the transmission of mechanical force (5). The proportion and structure of collagen fibrils are crucial for the function of tendons and changes to the structure or proportion may result in adaptation or injury (6). Collagen molecules are produced by tenocytes, and are subsequently aggregated end-to-end and side-to-side to produce collagen fibrils (3). During this self-assembly process, the diameter, length and organization of collagen fibrils is regulated by PGs (7). Decorin, biglycan and aggrecan are regarded as the principal PGs in tendons (8). Subsequently, collagen fibrils, organized into fibers, bundles, and fascicles, provide tendons with structural integrity and mechanical function (9).

As tendons transmit mechanical force, tenocytes are able to detect and convert mechanical signals through mechanotransduction mechanisms into cellular biological events, such as the synthesis and degradation of collagen (10). The tenocytes also secrete matrix metalloproteinases (MMPs) and tissue inhibitor of metalloproteinases (TIMPs) (10). Previous studies have indicated that a balance of MMPs and TIMPs regulates collagen synthesis and that an imbalance may result in collagen degradation $(11,12)$. Tsai et al (13) demonstrated that MMP-1 serves a major role in the degradation of Col I. MMP-1 is the predominant interstitial collagenase in humans; however, 
MMP-13 is predominant in rodents (14). TIMP-1 inhibits the MMP-mediated break-down of collagen matrix (15).

Furthermore, there is a vast difference in cellular biological responses to mechanical loading conditions according to the type, magnitude, frequency and intensity of these conditions $(16,17)$. Regarding intensity, it is recognized that moderate mechanical loading may induce positive effects on tendons, whereas strenuous mechanical loading may lead to injury (18). Despite essential progress in the field, the effect of different mechanical loading conditions on tendons remains undefined (19). The purpose of the present study was to investigate the effect of different exercise intensity, characterized by very distinct loading patterns, using treadmill running to assess the alternations of collagens, PGs, MMP-13 and TIMP-1 in the ECM of rat Achilles tendons, in order to gain insights to evaluate tendon patho-physiology.

\section{Materials and methods}

Experimental animals and exercise protocols. A total of 18 male Wistar rats (12-13 weeks old; weight, 200-250 g) were purchased from the Central Laboratory of Animal Science, Southern Medical University (Guangzhou, China) (NFYY-2012-056). These rats were randomly and evenly assigned to one of three groups as follows: i) sedentary control (CON, $n=6)$, ii) medium-intensity running ( $M I R, n=6)$ and iii) high-intensity running (HIR, $n=6)$. All animals were housed in cages with a controlled humidity (40-60\%) and temperature $\left(22 \pm 1^{\circ} \mathrm{C}\right)$ under a $12 \mathrm{~h}$ light/dark cycle, with ad libitum access to food and water. The protocol used in the present study was approved by the animal Ethics Committee of Nanfang Hospital, Southern Medical University (Guangzhou, China). The employed running protocol was described previously (20). Briefly, rats in the MIR and HIR groups were acclimatized to exercise for 1 week, which consisted of running on a treadmill at a speed of $10 \mathrm{~m} / \mathrm{min}$ for $30 \mathrm{~min} /$ day, 5 days/week. Subsequently, animals in the MIR and HIR groups were regularly trained for 8 weeks as described in Table I. Rats in the CON group were maintained in cages without any additional exercise. All experiments were conducted in accordance with the institutional guidelines for the care and use of experimental animals (20). At the end of the 8-week running program, all rats were sacrificed via carbon dioxide asphyxiation (flow rate: $30 \%$ volume/min) followed by cervical dislocation. Subsequently, Achilles tendon tissues were surgically excised and harvested from all rats.

Picrosirius red staining. Achilles tendon tissues from each group were obtained by surgery excision for histological staining, fixed in $10 \%$ buffered formalin $\left(4^{\circ} \mathrm{C}\right.$, overnight $)$ and embedded in paraffin. Samples were cut into 4-mm thick sections, deparaffinized and stained with 5\% Picrosirius red to highlight collagen fiber structure and improve its natural birefringence under a polarized light microscope at a magnification x20 (Axioskop 40 Pol; Carl Zeiss AG, Oberkochen, Germany).

Immunohistochemistry. Immunohistochemistry for Col I and Col III was performed as previously described (6). Briefly, Achilles tendon tissues were decalcified with $9 \%$ formic acid for $10 \mathrm{~min}$, washed with PBS for $1 \mathrm{~min}$ and embedded in
Table I. Treadmill running protocols for rats in the MIR and HIR groups, $(n=6)$. Completed 5 days a week for 8 weeks.

\begin{tabular}{lccc}
\hline Group & Speed, m/min & Inclination, & Duration, min \\
\hline MIR & 19.3 & 5 & 60 \\
HIR & 26.8 & 10 & 60 \\
\hline
\end{tabular}

MIR, medium-intensity running; HIR, high-intensity running.

paraffin. Then, 4-mm thick sections were cut and deparaffinized with xylene and different concentrations of ethanol (100, 95, 85 and $70 \%$ ). Endogenous peroxidase activity was quenched with $3 \%$ hydrogen peroxide for $20 \mathrm{~min}$ at room temperature. Antigen retrieval was performed with citric acid $(\mathrm{pH} 6.0)$ using a high pressure method (21). The citric acid buffer was preheated for $5 \mathrm{~min}$ in an autoclave and sections were boiled for $2 \mathrm{~min}$, followed by cooling for $20 \mathrm{~min}$. Sections were blocked with $5 \%$ normal bovine serum albumin (BSA; Merck KGaA, Darmstadt, Germany) for $20 \mathrm{~min}$ at room temperature and sections were incubated with specific primary antibodies at $4^{\circ} \mathrm{C}$ overnight. The mouse anti-rat primary antibodies anti-Col I (cat. no. ab6308) and anti-Col III (cat. no. ab6310) were diluted by 1:100 (Abcam, Cambridge, UK). Sections were subsequently incubated with horseradish peroxidase conjugated goat anti-mouse Imunoglobulin G (1:200; cat. no. sc2005; Santa Cruz Biotechnology, CA, USA) for $1 \mathrm{~h}$ at room temperature, developed with 3,3'-Diaminobenzidine tetrahydrochloride (DAKO; Agilent Technologies, Inc., Santa Clara, CA, USA) and counter-stained in hematoxylin. The primary antibody was replaced with $5 \% \mathrm{BSA}$ at $4^{\circ} \mathrm{C}$ overnight in the controls. To enable reproducibility and comparability, all incubation times and conditions were strictly controlled. The sections were examined under a color video camera attached to a H600L light microscope and image analysis system (Nikon Corporation, Tokyo, Japan). Images were captured using Image-Pro Plus version 6.0 software (Media Cybernetics, Inc., Rockville, MD, USA).

Transmission electron microscopy observation. Transmission electron microscopy (TEM) was completed as previously described (22). Achilles tendon tissues for TEM were fixed with $2.5 \%$ glutaraldehyde $/ 4 \%$ formaldehyde fixative for $2 \mathrm{~h}$ at $4^{\circ} \mathrm{C}$, post-fixed with $1 \%$ osmium tetroxide for $2 \mathrm{~h}$, dehydrated with ethanol (50, 70, 90 and 100\%; 20 min each step), embedded in Epon 812 and polymerized at $60^{\circ} \mathrm{C}$. Ultrathin (50-60 nm) cross-sections were observed at $60 \mathrm{kV}$ using a 7500 transmission electron microscope (Hitachi, Ltd., Tokyo, Japan) and digital images were captured at a magnification, x60,000 with a Megaview III digital camera (Olympus Soft Imaging Solutions GmbH, Münster, Germany). A total of 500 collagen fibrils in each group were randomly selected and diameters were measured using Scion Image 4.0 Software (Scion Corporation, Frederick, MD, USA).

Reverse transcription-quantitative polymerase chain reaction $(R T-q P C R)$. Achilles tendon tissues for RT-qPCR were frozen in liquid nitrogen and broken into pieces with a pestle and mortar. Subsequently, the fragments were mixed and placed in a vessel 
Table II. Primer sequence used in reverse transcription-quantitative polymerase chain reaction.

\begin{tabular}{lll}
\hline Primer & \multicolumn{1}{c}{ Forward } & \multicolumn{1}{c}{ Reverse } \\
\hline GAPDH & 5'-GGCACAGTCAAGGCTGAGAATG-3' & 5'-ATGGTGGTGAAGACGCCAGTA-3' \\
COL I & 5'-CATCGGTGGTACTAAC-3' & 5'-CTGGATCATATTGCACA-3' \\
COL III & 5'-GATGGCTGCACTAAAC-3' & 5'-CGAGATTAAAGCAAGAG-3' \\
Decorin & 5'-ATGATTGTCATAGAACTGGGC-3' & 5'-TTGTTGTTATGAAGGTAGAC-3' \\
Biglycan & 5'-TCTACATCTCCAAGAACCACCTGG-3' & 5'-GCTCTGGGCTCCTACTCCTT-3' \\
Aggrecan & 5'-ATCGTGGGCCGCCCTAGGCA-3' & 5'-TGGCCTTAGGGTTCAGAGGGG-3' \\
MMP-13 & 5'-TACAACTTGTTCCTTGTCGC-3' & 5'-CTGGGCCATAGAGAGACT-3' \\
TIMP-1 & 5'-CAGCGAGGAGTTTCTGG-3' & 5'-GGTAAACACTGTGCACCC-3'
\end{tabular}

GAPDH, glyceraldehyde-3-phosphate dehydrogenase; COL I, collagen type I; COL III, collagen type III; MMP-13, matrix metalloproteinase-13; TIMP-1, tissue inhibitors of metalloproteinase-1.

containing $1 \mathrm{ml}$ RNAiso Plus (Takara Biotechnology Co., Ltd., Dalian, China), followed by centrifugation at $13,362 \mathrm{x}$ for $15 \mathrm{~min}$ at $4^{\circ} \mathrm{C}$. Prior to mixing, $0.2 \mathrm{ml}$ chloroform (analytical pure) was added. The supernatant was removed following centrifugation at $13,362 \times \mathrm{g}$ for $15 \mathrm{~min}$ at $4^{\circ} \mathrm{C}, 500 \mathrm{ml}$ isopropanol was added and the samples were once again centrifuged at $13,362 \mathrm{x} \mathrm{g}$ for $15 \mathrm{~min}$ at $4^{\circ} \mathrm{C}$. The supernatant was discarded; $75 \%$ ethanol and $500 \mathrm{ml}$ diethyl pyrocarbonate (DEPC)-treated $\mathrm{H}_{2} \mathrm{O}$ were added. The samples were centrifuged at $4,547 \mathrm{x}$ g for $5 \mathrm{~min}$ at $4^{\circ} \mathrm{C}$. The supernatant was discarded and the pellet was air dried. Subsequently, $30 \mu \mathrm{l}$ of DEPC-treated $\mathrm{H}_{2} \mathrm{O}$ was added. Reverse transcription of the mRNA to template cDNA was completed using a PrimeScript RT reagent kit (Takara Biotechnology Co., Ltd.). The enzyme mix and RT primer mix were added to the mRNA sample and cDNA was generated by heating at $37^{\circ} \mathrm{C}$ for $15 \mathrm{~min}$ and $85^{\circ} \mathrm{C}$ for $5 \mathrm{sec}$. Quantitative PCR was performed using a 7500 Fast Real-Time PCR system (Applied Biosystems; Thermo Fisher Scientific, Inc., Waltham, MA, USA) and a SYBR Premix Ex Taq II kit (Takara Biotechnology Co., Ltd.); glyceraldehyde-3-phosphate dehydrogenase (GAPDH) was used as an endogenous reference and each sample was normalized to its GAPDH content. The PCR protocol used was as follows: $10 \mathrm{~min}$ heating at $95^{\circ} \mathrm{C}$, followed by 45 cycles at $95^{\circ} \mathrm{C}$ for $10 \mathrm{sec}$, $55^{\circ} \mathrm{C}$ for $15 \mathrm{sec}, 72^{\circ} \mathrm{C}$ for $30 \mathrm{sec}$. The mRNA expression of collagen (Col I and Col III), principal PGs (decorin, biglycan and aggrecan), MMP-13 and TIMP-1 in the Achilles tendon were detected via PCR. The sequences of PCR primers pairs (BioTeke Corporation, Beijing, China) are presented in Table II. The relative gene expression was calculated using the $2^{-\Delta \Delta C T}$ method (23). The assay was replicated in triplicate.

Statistical methods. Results are expressed as the mean \pm standard deviation. Statistical analysis was carried out using one-way analysis of variance and Tukey's test for post hoc analysis. Data analysis was performed using SPSS 16.0 (SPSS, Inc., Chicago, IL, USA) and $\mathrm{P}<0.05$ was considered to indicate a statistically significant difference.

\section{Results}

Picrosirius red staining. Following 8 weeks of treadmill running programs, Achilles tendons sections were stained with
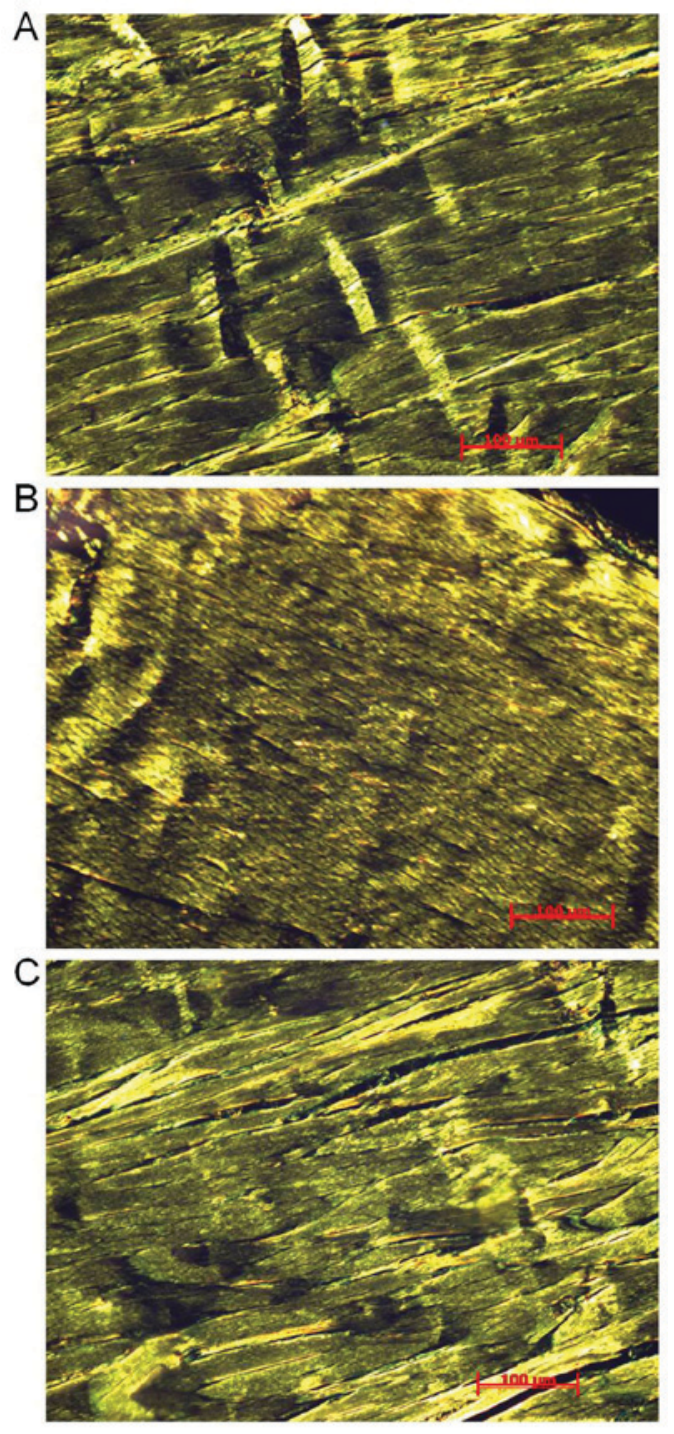

Figure 1. Representative histological sections of rat Achilles tendons. Sections, 4-mm thick stained with Picrosirius red, viewed under polarized light at magnification, $\mathrm{x} 20$ for the (A) control, (B) medium-intensity running and $(\mathrm{C})$ high-intensity running groups, $\mathrm{n}=6$ for all.

Picrosirius red to observe structural features of collagen fibrils using a polarized light microscope. Fig. 1 presents observed 

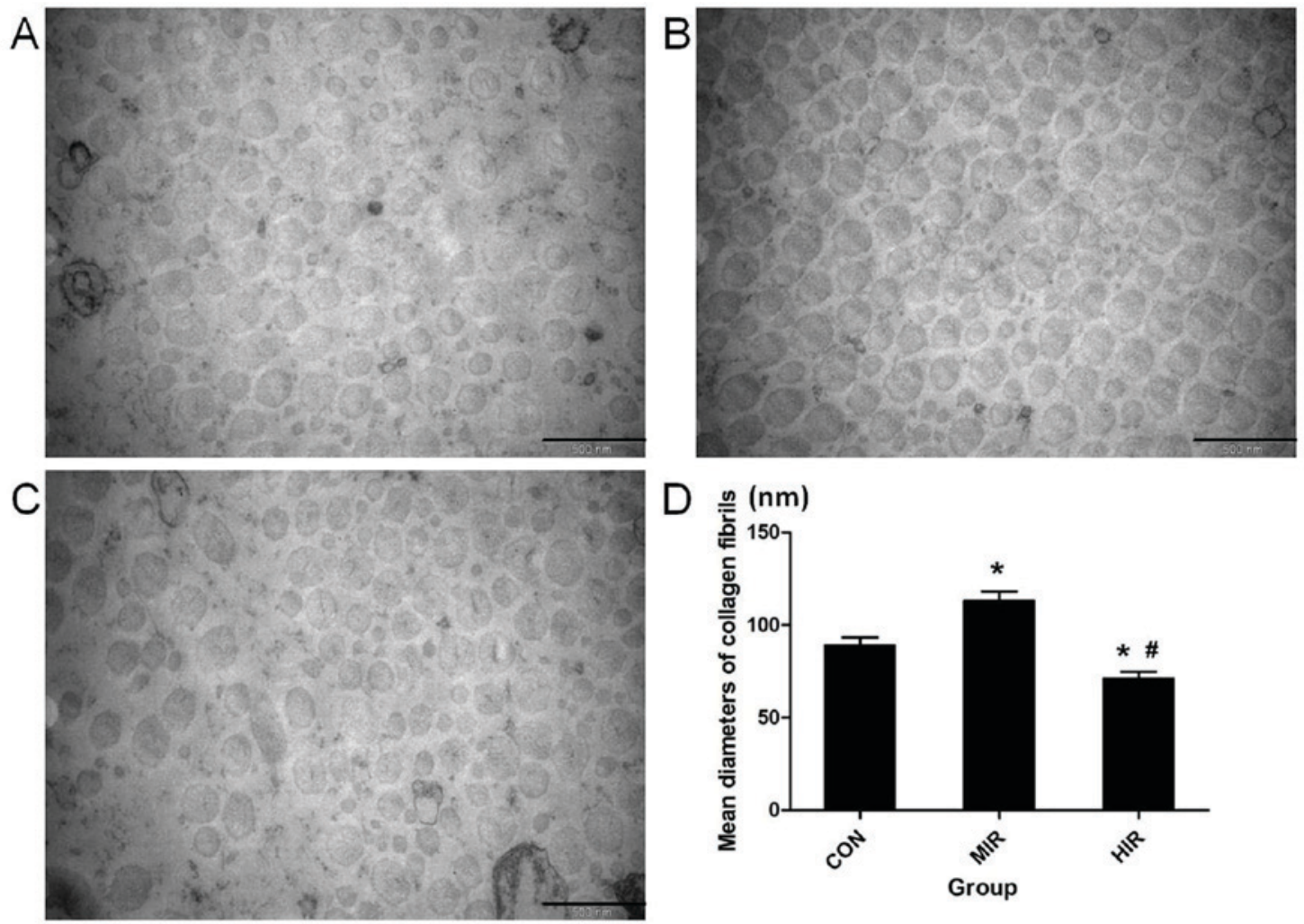

Figure 2. Mean diameter of collagen fibrils in rat Achilles tendons. (A) CON, (B) MIR and (C) HIR groups were evaluated using a transmission electron microscope and (D) the statistical analysis. Scale bar represents $500 \mathrm{~nm}$. Magnification, x60,000. ${ }^{*} \mathrm{P}<0.05$ vs. CON group; ${ }^{\mathrm{P}} \mathrm{P}<0.05$ vs. MIR group. CON, control; MIR, medium-intensity running; HIR, high-intensity running.

structural features in the three groups. Collagen fibers were organized in parallel with crimps in the CON group (Fig. 1A). Regularly and densely organized collagen fibrils were recorded in the MIR group (Fig. 1B). However, irregular and loosely organized collagen fibrils were observed in the HIR group (Fig. 1C).

TEM. Representative TEM images of the collagen fibrils in Achilles tendons in transverse section for each group are presented in Fig. 2; CON (Fig. 2A), MIR (Fig. 2B) and HIR (Fig. 2C). The diameter of collagen fibrils was calculated using Scion Image Software (Fig. 2D). Significantly thicker collagen fibrils were observed in the MIR group $(113 \pm 5.2 \mathrm{~nm})$ compared with the CON group $(89 \pm 4.3 \mathrm{~nm} ; \mathrm{P}<0.05)$. Furthermore, significantly thinner collagen fibrils were observed in the HIR group $(71 \pm 3.8 \mathrm{~nm})$ compared with the $\mathrm{CON}$ and MIR groups $(\mathrm{P}<0.05)$.

Immunohistochemistry. Fig. 3 presents representative immunostaining of $\mathrm{Col} \mathrm{I}$ in Achilles tendon sections in the CON (Fig. 3A), MIR (Fig. 3B) and HIR (Fig. 3C) groups. Immunohistological analysis for Col I was performed using Image-Pro Plus 6.0 software and is presented in Fig. 3D. The Col I content (image gray value) was significantly higher in the MIR group $(0.382 \pm 0.093)$ compared with the CON group $(0.267 \pm 0.026 ; \mathrm{P}<0.05)$ and was significantly lower in the HIR group $(0.203 \pm 0.084)$ compared with the CON or MIR groups $(\mathrm{P}<0.05)$.

Representative immunostaining of Col III in Achilles tendon sections is presented for each group in Fig. 4; CON (Fig. 4A), MIR (Fig.4B) and HIR (Fig.4C). Immunohistological analysis for Col III was completed using Image-Pro Plus 6.0 software (Fig. 4D). The Col III content (image gray value) was significantly higher in the HIR group $(0.189 \pm 0.075)$ compared with the CON $(0.161 \pm 0.018)$ or MIR $(0.166 \pm 0.044 ; \mathrm{P}<0.05)$ groups. No significant difference was observed between the CON and MIR groups.

$R T-q P C R$. Changes in the mRNA gene expression in rat Achilles tendons in CON, MIR and HIR groups is presented in Fig. 5. The expression of Col I was significantly upregulated in the MIR group compared with the $\mathrm{CON}$ group $(\mathrm{P}=0.024$; Fig. 5A). However, Col I expression was significantly downregulated in the HIR group compared with the CON or MIR groups ( $\mathrm{P}=0.037$ and $\mathrm{P}=0.019$, respectively; Fig. $5 \mathrm{~A})$. The expression of $\mathrm{Col}$ III was significantly upregulated in the HIR group when compared with the CON or MIR group $(\mathrm{P}=0.024$, $\mathrm{P}=0.047$, respectively; Fig. 5A). The changes in Col III mRNA gene expression were less pronounced in the MIR group in comparison with the CON group ( $\mathrm{P}=0.196$; Fig. 5A).

The expression of MMP-13 was significantly increased in the MIR and HIR groups in comparison with the CON group $(\mathrm{P}=0.028, \mathrm{P}=0.013$, respectively; Fig. 5B). However, a less pronounced change of MMP-13 mRNA expression was recorded in the HIR group in comparison with the MIR group $(\mathrm{P}=0.075$; Fig. 5B). The expression of TIMP-1 was increased in the MIR group compared with the $\mathrm{CON}$ group $(\mathrm{P}=0.018$; Fig. 5B), but significantly decreased in the HIR group compared with the CON or MIR groups $(\mathrm{P}=0.033, \mathrm{P}=0.027$, respectively). 

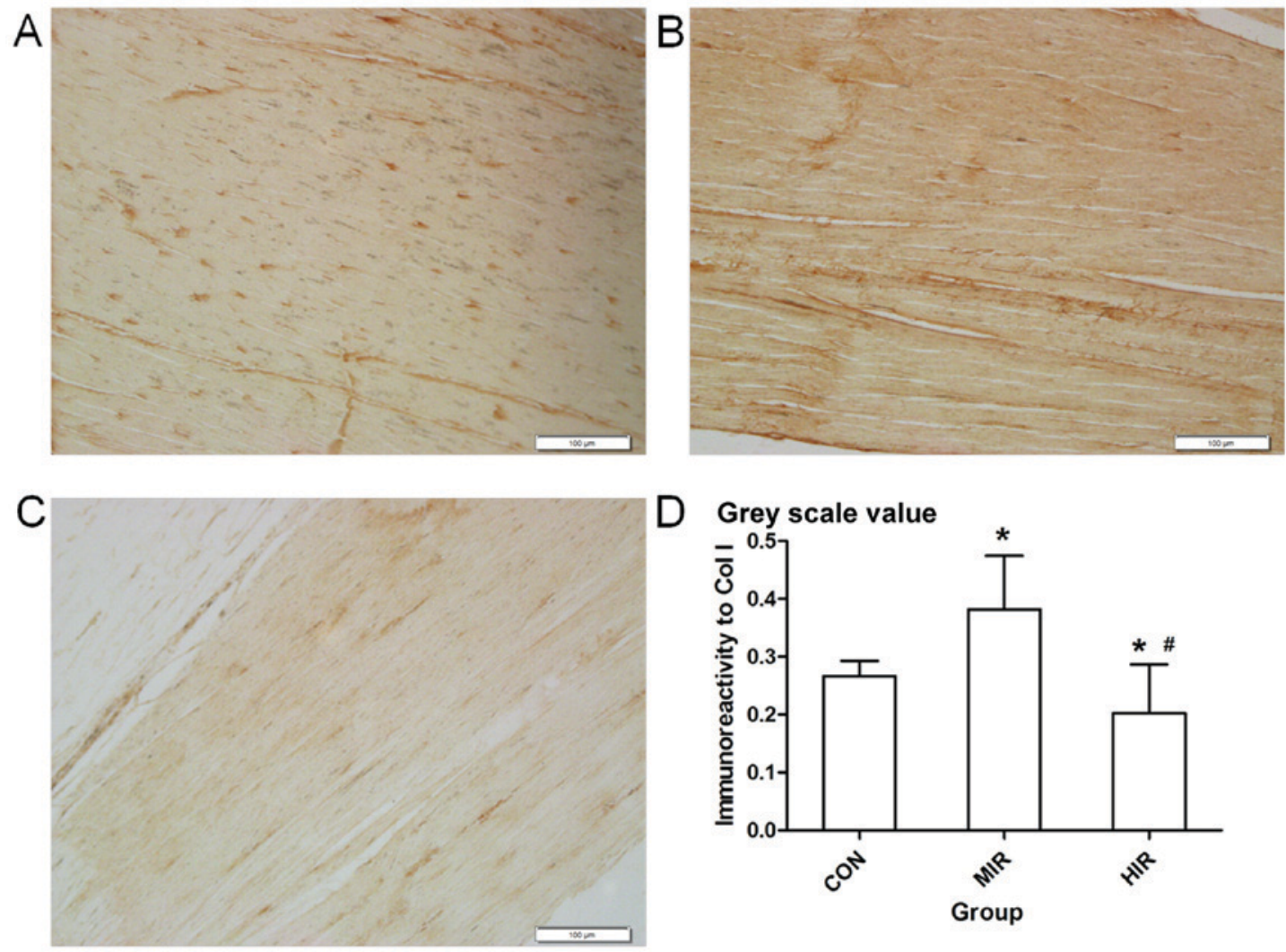

\section{Grey scale value}

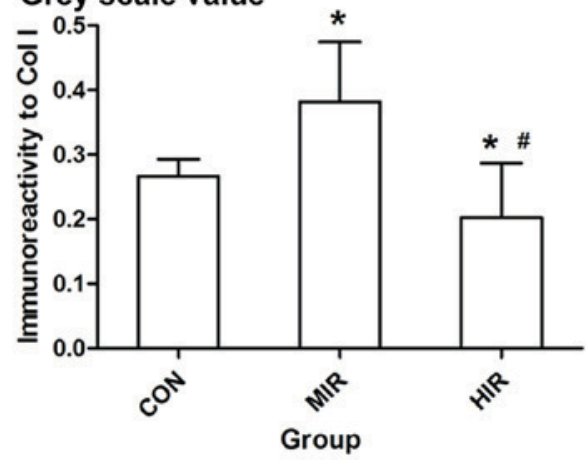

Figure 3. Representative images of the immunohistochemical staining, developed with 3,3'-Diaminobenzidine tetrahydrochloride and counter stained with hematoxylin for $\mathrm{Col} \mathrm{I}$ in the rat Achilles tendon. (A) CON, (B) MIR and (C) HIR groups and (D) the statistical analysis. Scale bar represents $100 \mu \mathrm{m}$. ${ }^{\text {"P }}<0.05$ vs. CON group; ${ }^{*} \mathrm{P}<0.05$ vs. MIR group. Col I, collagen type I; CON, control; MIR, medium-intensity running; HIR, high-intensity running.
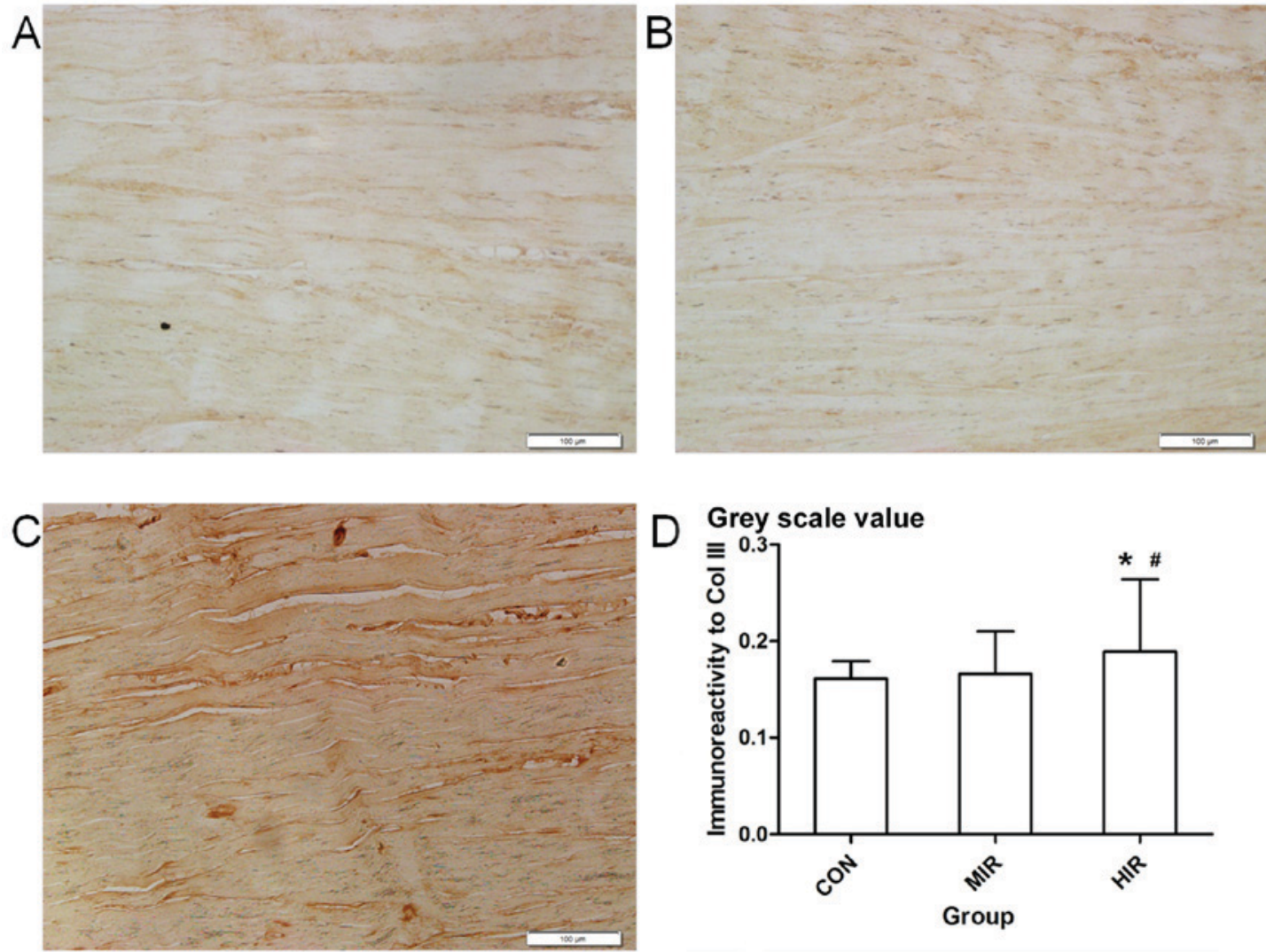

Figure 4. Representative images of immunohistochemical staining, developed with 3,3'-Diaminobenzidine tetrahydrochloride and counter stained with hematoxylin for Col III in rat Achilles tendon. (A) CON, (B) MIR and (C) HIR groups, and (D) the statistical analysis. Scale bar represents $100 \mu \mathrm{m}$. ${ }^{*} \mathrm{P}<0.05 \mathrm{vs}$. CON group; ${ }^{~} \mathrm{P}<0.05$ vs. MIR group. Col III, collagen type III; CON, control; MIR, medium-intensity running; HIR, high-intensity running. 
A

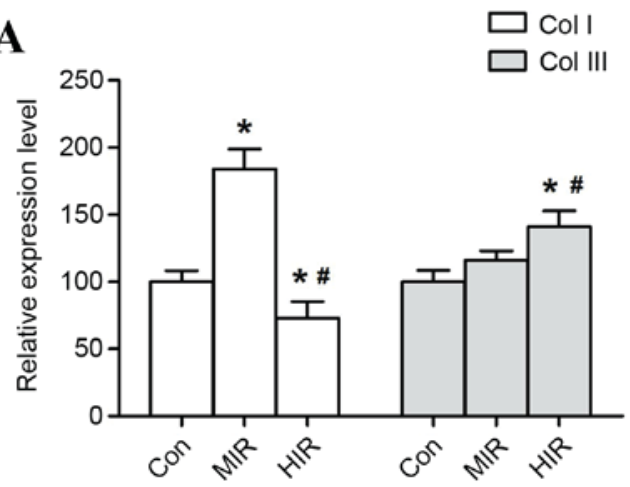

B

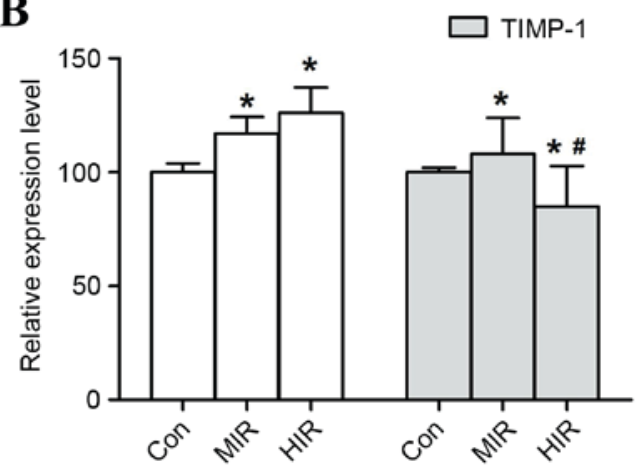

C

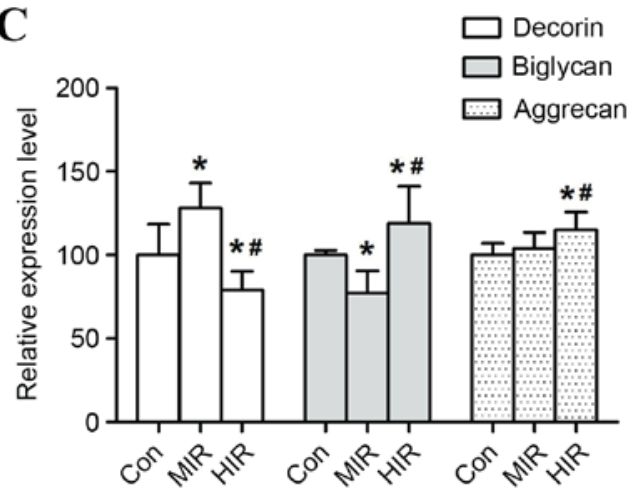

Figure 5. mRNA expression in rat Achilles tendons. mRNA expression of (A) Col I and Col III, (B) MMP-13 and TIMP-1 and (C) decorin, biglycan and aggrecan in rat Achilles tendons following MIR and HIR were determined by reverse transcription-quantitative polymerase chain reaction. GAPDH was used as an endogenous reference. Data are presented as mean \pm standard deviation, $\mathrm{n}=3,{ }^{*} \mathrm{P}<0.05$ vs. $\mathrm{CON} ;{ }^{*} \mathrm{P}<0.05$ vs. MIR group. Col I, collagen type I; Col III, collagen type III; MMP-13, metalloproteinase-13; TIMP-1, tissue inhibitor of metalloproteinases-1; MIR, medium-intensity running; HIR, high-intensity running.

Additionally, decorin expression was significantly higher in MIR group than that in CON group $(\mathrm{P}=0.034)$, whereas it was significantly lower in HIR group compared with the CON or MIR groups $(\mathrm{P}=0.039, \mathrm{P}=0.032$, respectively; Fig. $5 \mathrm{C})$. Conversely, biglycan expression was significantly lower in MIR group than in the $\mathrm{CON}$ group $(\mathrm{P}=0.022)$, while significantly higher in the HIR group compared with the CON or MIR groups $(\mathrm{P}=0.038, \mathrm{P}=0.027$, respectively; Fig. $5 \mathrm{C})$. Aggrecan expression was significantly higher in the HIR group compared with the $\mathrm{CON}$ or MIR groups $(\mathrm{P}=0.017, \mathrm{P}=0.013$, respectively). The change in mRNA gene expression of aggrecan was less significant in the MIR group in comparison with the CON group ( $\mathrm{P}=0.073$; Fig. 5C).

\section{Discussion}

In the present study, a running treadmill model at different speeds and inclinations was used to represent distinct exercise intensity and differentiate moderate and strenuous exercise. The results of the current study indicate that the crucial component, Col I was significantly increased in the MIR group compared with that in CON group $(\mathrm{P}<0.05)$. However, Col I was significantly decreased in the HIR group compared with the CON or MIR groups $(\mathrm{P}<0.05)$. Therefore demonstrating that synthesis or degradation of Col I occurred following 8 weeks of treadmill running. To evaluate the metabolism of collagen, the expression of MMP-13 and TIMP-1 was examined. Data from the present study indicated that the expression of MMP-13 and TIMP-1 was significantly increased in the MIR group $(\mathrm{P}<0.05)$. However, the expression of MMP-13 increased and the expression of TIMP-1 decreased in the HIR group. The aforementioned results suggested that MMP-13 and TIMP-1 were balanced and collagen synthesis occurred following MIR, but were imbalanced following HIR, which may lead to collagen degradation. These findings suggest that moderate exercise induced a balanced expression of MMPs and TIMPs, allowing collagen synthesis to improve mechanical strength for transmission. However, strenuous exercise induced an imbalance, leading to collagen degradation which may weaken mechanical strength and increase the risk of damage (24).

In addition, it should be noted that the level of Col III was increased in the HIR group. Col III has been reported to intercalate into the $\mathrm{Col}$ I fibrils and produce smaller, less organized fibrils (24). It was also regarded as an immature collagenous matrix (25). This may in accordance with the results of TEM in the present study, in which more mature and large diameter fibrils were observed in the MIR group but in the HIR group more immature and small diameter fibrils were observed (Fig. 2).

It has been indicated that PGs serve a vital role in the self-assembly process of collagen fibrils (26). The results of the present study revealed that decorin expression was higher in the MIR group and lower in the HIR group. This supports the observations made following Picrosirius red staining, in which regular collagen fibril arrangement in the MIR group was observed, whereas irregular arrangement was observed in the HIR group. Decorin has been reported to regulate the fibril diameter and help organize and orientate the collagen fibrils in tendons (27). However, a converse pattern of changes was identified in biglycan expression among the three groups. This is potentially due to high homology and co-expression of biglycan and decorin (28). Therefore, they may share common functions and partially compensate for each other's functions. Additionally, the findings of the current study indicated that aggrecan expression was higher in the HIR group. Aggrecan is located between adjacent collagen fiber bundles and increases the tendon hydration and fibril separation (29). This may explain the presence of more spaces between and in collagen fibril bundles observed in the HIR group.

However, the present study was limited by the use of only one time point ( 8 weeks) and the difference between rodent 
and human MMP expression. In addition, the alterations of a number of major molecules in ECM were observed, while other molecules may change and serve important roles in tendon patho-physiology.

In conclusion, the current study demonstrated a significant intensity-specific effect following treadmill running on the rat Achilles tendon. These results suggest that moderate exercise may induce increased collagen synthesis and organize regular and large collagen fibers, thus benefiting the Achilles tendon. Nevertheless, overuse may result in collagen degradation and disturbance, which is predisposed to injury. However, further studies with more time points and time frames should be completed to validate the findings.

\section{Acknowledgements}

The authors of the present study gratefully acknowledge Mr PR Zhao for technical assistance. The current study was supported by Natural Science Foundation of China (grant no. 81371686 and 81572219) and Guangdong Natural Science Foundation (grant no. S20140006946).

\section{Competing interests}

The authors declare that they have no competing interests.

\section{References}

1. Lin TW, Cardenas L and Soslowsky LJ: Biomechanics of tendon injury and repair. J Biomech 37: 865-877, 2004.

2. Heinemeier KM and Kjaer M: In vivo investigation of tendon responses to mechanical loading. J Musculoskelet Neuronal Interact 11: 115-123, 2011.

3. Juneja SC and Veillette C: Defects in tendon, ligament, and enthesis in response to genetic alterations in key proteoglycans and glycoproteins: A review. Arthritis 2013: 154812, 2013.

4. Amiel D, Frank C, Harwood F, Fronek J and Akeson W: Tendons and ligaments: A morphological and biochemical comparison. J Orthop Res 1: 257-265, 1984.

5. Franchi M, Torricelli P, Giavaresi G and Fini M: Role of moderate exercising on Achilles tendon collagen crimping patterns and proteoglycans. Connect Tissue Res 54: 267-274, 2013.

6. Lui PP, Chan LS, Lee YW, Fu SC and Chan KM: Sustained expression of proteoglycans and collagen type III/type I ratio in a calcified tendinopathy model. Rheumatology (Oxford) 49: 231-239, 2010

7. Reese SP, Underwood CJ and Weiss JA: Effects of decorin proteoglycan on fibrillogenesis, ultrastructure, and mechanics of type I collagen gels. Matrix Biol 32: 414-423, 2013.

8. Rees SG, Flannery CR, Little CB, Hughes CE, Caterson B and Dent CM: Catabolism of aggrecan, decorin and biglycan in tendon. Biochem J 350: 181-188, 2000.

9. Wang JH: Mechanobiology of tendon. J Biomech 39: 1563-1582, 2006.

10. Wang JH, Thampatty BP, Lin JS and Im HJ: Mechanoregulation of gene expression in fibroblasts. Gene 391: 1-15, 2007.

11. Dalton S, Cawston TE, Riley GP, Bayley IJ and Hazleman BL Human shoulder tendon biopsy samples in organ culture produce procollagenase and tissue inhibitor of metalloproteinases. Ann Rheum Dis 54: 571-577, 1995.
12. Thampatty BP, $\mathrm{Li} \mathrm{H}, \mathrm{Im} \mathrm{HJ}$ and Wang JH: EP4 receptor regulates collagen type-I, MMP-1, and MMP-3 gene expression in human tendon fibroblasts in response to IL-1 beta treatment. Gene 386: 154-161, 2007.

13. Tsai WC, Hsu CC, Chang HN, Lin YC, Lin MS and Pang JH: Ibuprofen upregulates expressions of matrix metalloproteinase-1, $-8,-9$, and -13 without affecting expressions of types I and III collagen in tendon cells. J Orthop Res 28: 487-491, 2010.

14. Wisløff $\mathrm{U}$, Helgerud $\mathrm{J}$, Kemi OJ and Ellingsen $\mathrm{O}$ : Intensity-controlled treadmill running in rats: $\operatorname{VO}(2 \max )$ and cardiac hypertrophy. Am J Physiol Heart Circ Physiol 280: H1301-H1310, 2001.

15. Brew $\mathrm{K}$ and Nagase $\mathrm{H}$ : The tissue inhibitors of metalloproteinases (TIMPs): An ancient family with structural and functional diversity. Biochim Biophys Acta 1803: 55-71, 2010.

16. Firth EC: The response of bone, articular cartilage and tendon to exercise in the horse. J Anat 208: 513-526, 2006.

17. Magnusson SP, Hansen P and Kjaer M: Tendon properties in relation to muscular activity and physical training. Scand J Med Sci Sports 13: 211-223, 2003.

18. Jhingan S, Perry M, O'Driscoll G, Lewin C, Teatino R, Malliaras P, Maffulli N and Morrissey D: Thicker Achilles tendons are a risk factor to develop Achilles tendinopathy in elite professional soccer players. Muscles Ligaments Tendons J 1: $51-56,2011$

19. Zhang $\mathbf{J}$ and Wang $\mathrm{JH}$ : The effects of mechanical loading on tendons-an in vivo and in vitro model study. PLoS One 8: e71740, 2013.

20. Ni GX, Liu SY, Lei L, Li Z, Zhou YZ and Zhan LQ: Intensity-dependent effect of treadmill running on knee articular cartilage in a rat model. Biomed Res Int 2013: 172392, 2013.

21. Norton AJ, Jordan S and Yeomans P: Brief, high-temperature heat denaturation (pressure cooking): A simple and effective method of antigen retrieval for routinely processed tissues. J pathol 173: 371-379, 1994.

22. Dunkman AA, Buckley MR, Mienaltowski MJ, Adams SM, Thomas SJ, Satchell L, Kumar A, Pathmanathan L, Beason DP, Iozzo RV, et al: The tendon injury response is influenced by decorin and biglycan. Ann Biomed Eng 42: 619-630, 2014.

23. Livak KJ and Schmittgen TD: Analysis of relative gene expression data using real-time quantitative PCR and the 2(-Delta Delta C(T)) method. Methods 25: 402-408, 2001

24. Tan SC and Chan O: Achilles and patellar tendinopathy: Current understanding of pathophysiology and management. Disabil Rehabil 30: 1608-1615, 2008.

25. Fu SC, Wong YP, Cheuk YC, Lee KM and Chan KM: TGF-beta1 reverses the effects of matrix anchorage on the gene expression of decorin and procollagen type I in tendon fibroblasts. Clin Orthop Relat Res: 226-232, 2005.

26. Kalamajski S and Oldberg A: The role of small leucine-rich proteoglycans in collagen fibrillogenesis. Matrix Biol 29: 248-253, 2010

27. Scott JE: Elasticity in extracellular matrix 'shape modules' of tendon, cartilage, etc. A sliding proteoglycan-filament model. J Physiol 553: 335-343, 2003.

28. Säämänen AM, Salminen HJ, Rantakokko AJ, Heinegård D and Vuorio EI: Murine fibromodulin: cDNA and genomic structure, and age-related expression and distribution in the knee joint. Biochem J 355: 577-585, 2001.

29. Smith MM, Sakurai G, Smith SM, Young AA, Melrose J, Stewart CM, Appleyard RC, Peterson JL, Gillies RM, Dart AJ, et al: Modulation of aggrecan and ADAMTS expression in ovine tendinopathy induced by altered strain. Arthritis Rheum 58: 1055-1066, 2008. 